- Provides a review of Australian indigenous health and its relationship to oral health.

- Improves awareness of Australian indigenous health among UK dentists.

- Helps raise the profile of working overseas, particularly within Australia.

\title{
Improving cross-cultural awareness. A review of Australian indigenous health for UK dentists
}

\author{
H. Popat ${ }^{1}$ and J. Dinnage ${ }^{2}$
}

Objective The aim of this paper is to give an insight into the unique health issues faced by the indigenous population of Australia and their direct relationship to oral health, to help overseas dentists wishing to work in Australia to treat these groups of patients successfully and effectively. This applies equally to indigenous Australians living in remote Australia as well as to those living in general suburbia.

Data sources $A$ Medline ${ }^{\circledR}$ search was carried out for any articles in dental and medical journals pertaining to Australian indigenous health and Australian oral health.

Data selection Wherever possible, articles cited were obtained in full and where this was not possible, abstracts were obtained. Where no abstract was available, the article was not considered for evaluation. Data extraction Articles were reviewed by a single observer and were subject to meeting inclusion criteria indicated in the review.

Data synthesis Articles were divided into historical, diet, oral health and general health categories. Subjective descriptions were then made. Conclusions The adoption of a 'westernised' diet by the Australian indigenous community has placed them as a high risk population for dental caries and periodontal disease. They also show some of the highest rates in the world for Non-Insulin Dependent Diabetes Mellitus (NIDDM) and Rheumatic Fever. The UK dentist should be aware of these health issues and their relationship to indigenous oral health before embarking on work within Australia.

\section{INTRODUCTION}

With mutual recognition still current practice in Australia, dental graduates from the United Kingdom are able to work freely in most parts of Australia and New Zealand.

Although the fundamental practices of dentistry are universal throughout the world, patients are not. The majority of non-Aus-

${ }^{1}$ Specialist Registrar, Department of Orthodontics, University Dental Hospital, Heath Park, Cardiff, CF14 4XY; ${ }^{2}$ General Dental Practitioner, Malpas Dental Practice, Malpas Road, Newport NP20 6WE

Correspondence to: Dr Hashmat Popat

Email: popath@cardiff.ac.uk

\section{Refereed paper}

Accepted 28 September 2005

DOI: $10.1038 /$ sj.bdj.4813773

$\odot$ British Dental Journal 2006; 201: 37-42 tralian dental graduates will have had little or no experience of treating patients from an Australian indigenous background. The indigenous community is a unique part of the Australian population which presents with specific cultural, social, economic and health problems. It is imperative for the dental graduate who is considering work in Australia to have an understanding of the issues faced by the indigenous population and their relationship to oral health. This will allow dental treatment to be carried out both effectively and successful.

\section{BACKGROUND}

The Aboriginal and Torres Strait Islander population of Australia is estimated to be $2.4 \%$ of the total population. ${ }^{1}$

International media reporting of Australia's indigenous peoples often tends to focus on remote or nomadic communities, perpetuating narrow and often stereotyped perceptions of contemporary Aboriginal and Torres Strait Islander lifestyles. The reality is that indigenous peoples live in every state and territory of Australia and in highly urbanised environments, as well as relatively remote areas ( Figs 1 and 2).

\section{Diet and oral health}

Examination of Aboriginal skulls predating European colonisation (1788) found that dental caries was very rare. ${ }^{2}$ The traditional nomadic diet was low in sugar and contained a healthy balance of nutrients. This is supported by current data that state dental caries incidence as being very low in Aboriginal communities due to a more traditional way of life. ${ }^{3}$

Since colonisation however, the switch to a more 'western' diet has significantly increased caries incidence. ${ }^{4}$ Larger quantities of white flour, sugar and tea are consumed and fewer fresh fruits and vegetables. Rural and remote communities tend to purchase cheaper food products that contain large amounts of refined carbohydrates but are deficient in vitamins and protein. ${ }^{3-4}$ In 1982, an estimate of the sugar consumption of Groote Eylanders (a remote Aboriginal community) was $90 \mathrm{~kg}$ per person per year which was twice the Australian average. ${ }^{5}$ In 1975, a similar study at Echo Island showed that there was over $3 \mathrm{~kg}$ of sugar per person per week being purchased which, at the time, was almost three and half times the national average. ${ }^{6}$ 


\section{RESEARCH}

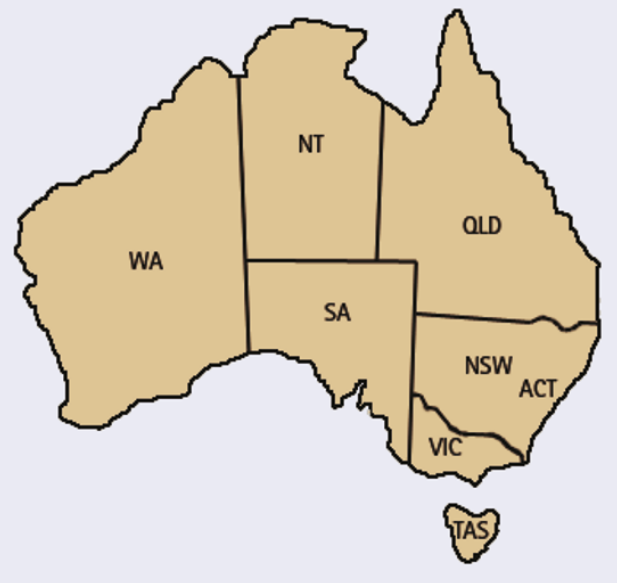

Fig. 1 State map of Australia

Children are particularly at risk from the increase in refined sugar intake. While the dental health of non-indigenous children has generally improved since the introduction of the School Dental Scheme in 1977 and fluoridation of water supplies (excluding Brisbane), the dental health of indigenous children appears to have deteriorated. ${ }^{7}$ Findings show however, that not only do indigenous children have significantly more decayed teeth and higher aggregate caries experience than non-indigenous children, but they also have poor access to treatment, longer periods between visits for dental care and a lack of community-based preventive measures. Data for dmfts and DMFTs are generally based on individual states and collected by different authorities so reports are not strictly comparable.

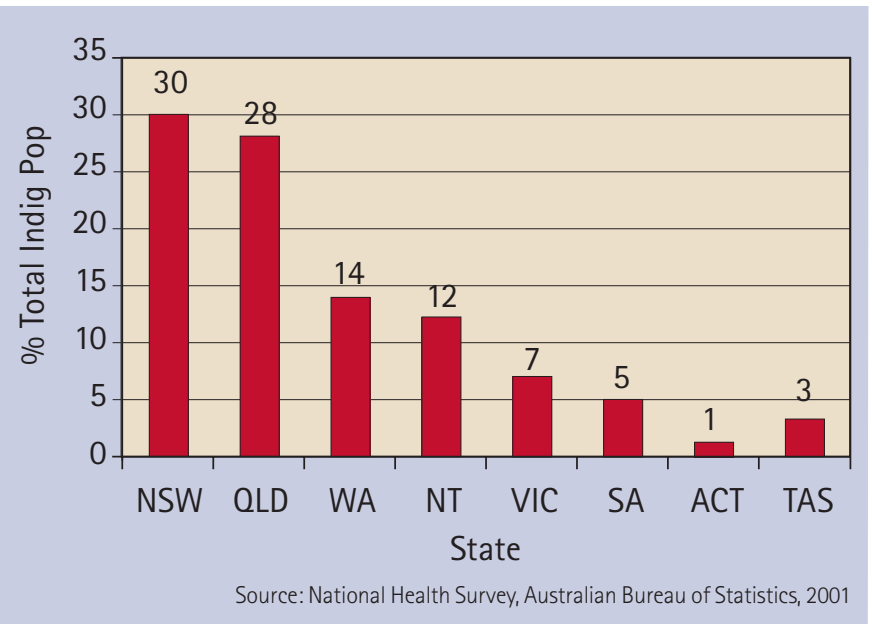

Fig. 2 Percentage spread of indigenous population across Australia by state

\section{Child dental health in New South Wales (NSW) and Northern} Territory (NT)

Indigenous children aged four to 12 years had a higher mean number of both deciduous and permanent teeth with decay experience than non-indigenous children in 2000 (Table 1).

The mean number of deciduous teeth with decay experience was higher for indigenous children than non-indigenous children across all ages from four to 11 years (Table 1).

The mean number of permanent teeth with decay experience for indigenous children ranged from 0.32 for six-year-olds to 2.92 for 12 -year-olds, compared with 0.27 for six-year-olds and 2.68 for 12 -year-olds for non-indigenous children (Table 1).

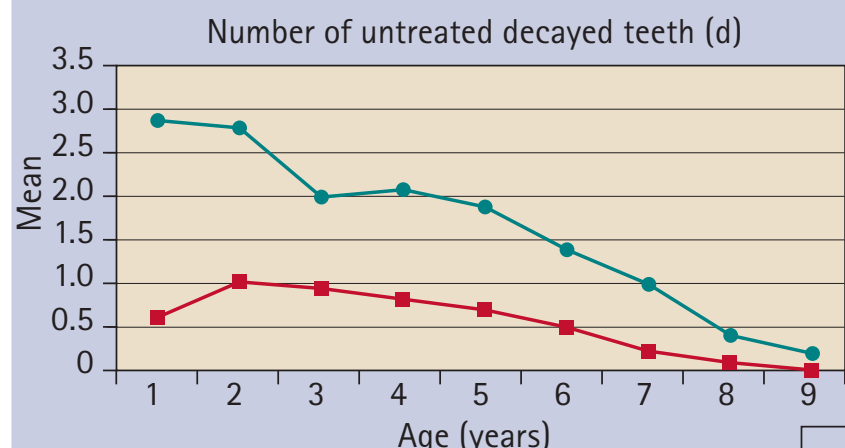

$\longrightarrow$ Indig
$\rightarrow-$ Non-indig

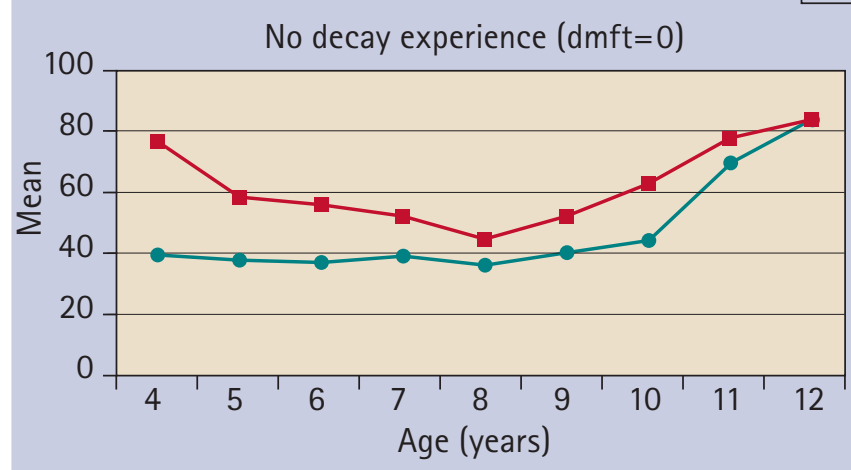

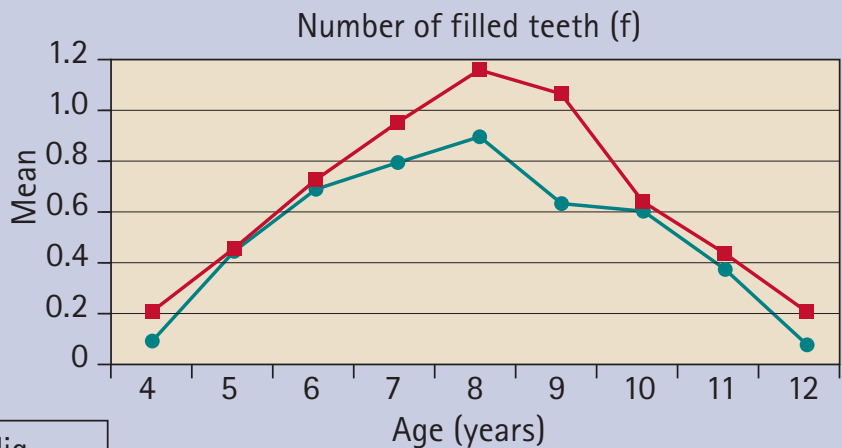

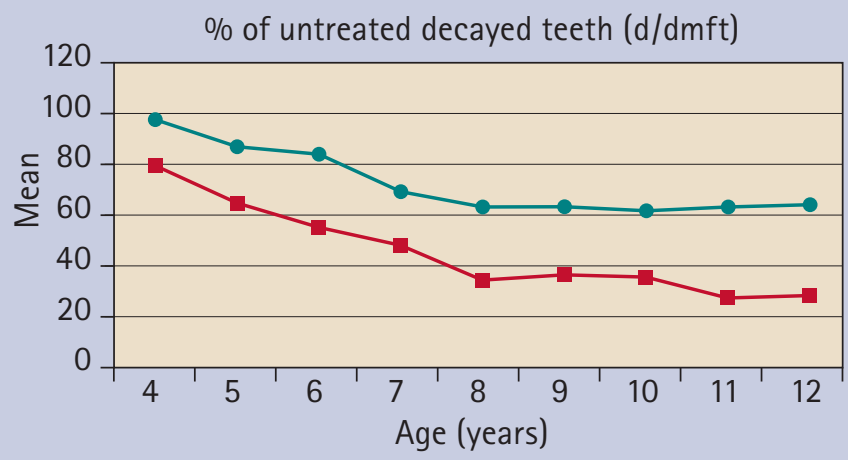

Source: Armfield J M, Roberts-Thomson K F. Child Dental Health Survey, Northern Territory 2001, AlHW and the University of Adelaide.

Fig. 3 Deciduous dental caries experience of children aged 4-12 years in the NT, by indigenous status, 2001 
RESEARCH

\begin{tabular}{|c|c|c|c|c|c|c|}
\hline & \multicolumn{2}{|c|}{ Metropolitan } & \multicolumn{2}{|c|}{ Regional \&t Remote } & \multicolumn{2}{|l|}{ Total } \\
\hline & Indig & Non-Indig & Indig & Non-Indig & Indig & Non-Indig \\
\hline \multicolumn{7}{|c|}{ Mean dmft score (for deciduous teeth) } \\
\hline 4 years & 7.11 & 5.04 & 8.10 & 6.31 & 7.73 & 5.02 \\
\hline 5 years & 5.62 & 4.60 & 5.60 & 5.30 & 5.62 & 4.75 \\
\hline 6 years & 4.80 & 4.44 & 5.43 & 5.17 & 5.50 & 4.59 \\
\hline 7 years & 4.62 & 3.53 & 4.67 & 4.12 & 4.65 & 3.63 \\
\hline 8 years & 5.38 & 3.36 & 4.18 & 3.76 & 4.42 & 3.44 \\
\hline 9 years & 3.16 & 2.84 & 3.67 & 3.15 & 3.40 & 2.90 \\
\hline 10 years & 2.50 & 2.10 & 2.23 & 2.84 & 2.34 & 2.25 \\
\hline 11 years & 0.80 & 1.10 & 1.41 & 1.39 & 1.22 & 1.15 \\
\hline 12 years & 0.50 & 0.47 & 0.49 & 0.60 & 0.49 & 0.49 \\
\hline \multicolumn{7}{|c|}{ Mean DMFT score (for permanent teeth) } \\
\hline 4 years & - & - & - & 0.10 & - & 0.02 \\
\hline 5 years & - & 0.04 & - & 0.05 & - & 0.05 \\
\hline 6 years & 0.28 & 0.27 & 0.38 & 0.24 & 0.32 & 0.27 \\
\hline 7 years & 0.66 & 0.73 & 0.59 & 0.81 & 0.65 & 0.75 \\
\hline 8 years & 0.37 & 0.87 & 1.59 & 1.09 & 1.34 & 0.92 \\
\hline 9 years & 1.14 & 1.01 & 1.62 & 0.95 & 1.49 & 1.00 \\
\hline 10 years & 1.58 & 1.39 & 2.99 & 1.35 & 2.43 & 1.38 \\
\hline 11 years & 1.85 & 1.78 & 2.33 & 2.16 & 2.26 & 1.85 \\
\hline 12 years & 2.00 & 2.67 & 3.01 & 2.58 & 2.92 & 2.68 \\
\hline
\end{tabular}

\section{Child dental health in the NT}

In 2001, indigenous children had a higher mean number of deciduous teeth with untreated decay than non-indigenous children in the NT (Fig. 3).

The proportion of indigenous children caries-free in their deciduous teeth was lower than the proportion for non-indigenous children (Fig. 3).

Indigenous children of all age groups had a higher ratio of untreated deciduous decayed teeth to the total count of decayed, missing, and filled teeth (d/dmft). This shows that indigenous children had a greater unmet need for treatment than non-indigenous children (Fig. 3).

\section{DIET}

The diet-related problem of the Australian indigenous population is multi-factorial. Not only is the high sugar consumption a problem, malnutrition has also been found to be relatively common. The 1993 Regional Health Survey in Queensland found that 23\% of people aged 15 years and above reported that in the previous 12 months, they had run out of food with no money to buy any more. ${ }^{9}$ A study in the rural south of Western Australia found that inadequate calcium, vitamin $\mathrm{C}$ and riboflavin intakes were common in rural Aboriginal communities. ${ }^{10}$ Another study reported frequent under-nutrition in young children in the Kimberley region (3000km north of Perth) due to a diet high in fat and sugar and low in calcium, fresh fruits and vegetables. ${ }^{11}$ Deficiencies in vitamin $\mathrm{C}$ from a lack of fresh fruit can cause conditions such as scurvy which can have detrimental effects on the periodontium. ${ }^{12}$
Obesity has also become a problem in adults. Rates of overweight and obese adults were over one and half times greater for Aboriginal and Torres Strait Islanders than for all Queenslanders. ${ }^{13}$ Being overweight or obese has been associated with an increased mortality and morbidity from a number of conditions including coronary heart disease, hypertension, non-insulin dependent diabetes and degenerative bone disease.

The high prevalence of poor nutrition among indigenous communities combined with poor oral hygiene has had the potential to significantly increase periodontal disease and the rate of dental caries among indigenous communities within Australia.

\section{MEDICAL CONDITIONS AND ORAL HEALTH}

\section{Diabetes}

Non-insulin dependent diabetes mellitus (NIDDM) is a form of diabetes mellitus that develops gradually in adults. It can be precipitated by obesity or severe stress or menopause as well as genetic factors. NIDDM or Type II diabetes is characterised by hyperglycaemia due to impaired insulin secretion, insulin resistance in muscle and non suppressible hepatic glucose production. It can usually be controlled by diet and hypoglycaemic agents without injections of insulin. ${ }^{14}$

The associations between oral health and diabetes are well documented. Studies have shown that both indigenous and nonindigenous patients with NIDDM experience an increased level of periodontal disease and dental caries. ${ }^{15-17}$ Patients with a longer duration of diabetes and poorly controlled diabetes are 
particularly at risk from severe periodontitis and tooth loss. ${ }^{18}$ Other studies have positively tested the hypothesis that persons with NIDDM have a greater risk of more severe alveolar bone loss than those without NIDDM. In addition to this, results suggest an NIDDM-associated increased rate of alveolar bone loss progression over time. ${ }^{19}$ NIDDM has also been found to be a risk factor for mucosal diseases such as xerostomia, candida albicans, glossodynia and even temperomandibular joint dysfunction syndrome. ${ }^{20-21}$

The traditional hunter-gatherer lifestyle of Aboriginal people was characterised by high physical activity and a diet of low energy density (low fat, high fibre) which promoted the maintenance of a very lean body weight and minimised insulin resistance. In contrast, the western lifestyle adopted by some indigenous communities is characterised by a reduced physical activity and an energy dense diet high in refined carbohydrate and fat that promotes obesity and maximises insulin resistance.

Although epidemiological studies and statistical analysis of indigenous communities vary between papers, studies show that diabetes incidence rates in Australian aboriginal people are among the highest in the world. It is Type II, or NIDDM that is the most common form suffered by indigenous communities. ${ }^{22}$

Many studies have shown that the incidence of NIDDM is much higher among indigenous communities within Australian than among the non-indigenous population. ${ }^{22}$ While between 2-5\% of the non-aboriginal population in Australia has NIDDM, the prevalence of diabetes among Australian aboriginal populations is much higher with rates quoted between 15 and $30 \% .{ }^{23}$ The overall prevalence of NIDDM among Australians aged greater than 25 years of age is 7.5\%. Prevalence rates up to four times higher have been reported for indigenous communities - 29.6\% in over 35 year olds in an aboriginal community in central Australia. ${ }^{24}$ This study also found the incidence in the below 35 year old age group to still be relatively high at 5.3\%. Another study used community based screening to find that obesity rates for Torres Strait Islanders were three times higher and diabetes rates six times higher than for other Australians ${ }^{25}$ (Fig. 4).

The high rate of NIDDM within the indigenous community of Australia is having a significant impact on the risk of periodontal disease and caries. People living in remote areas are now becoming edentulous and this is mostly from diabetes-related periodontal disease. ${ }^{26}$ Unfortunately, with the strongest non-metabolic risk factor for NIDDM being obesity and the prevalence of obesity related in part to an inappropriate diet high in refined carbohydrate, a vicious circle may ensue.

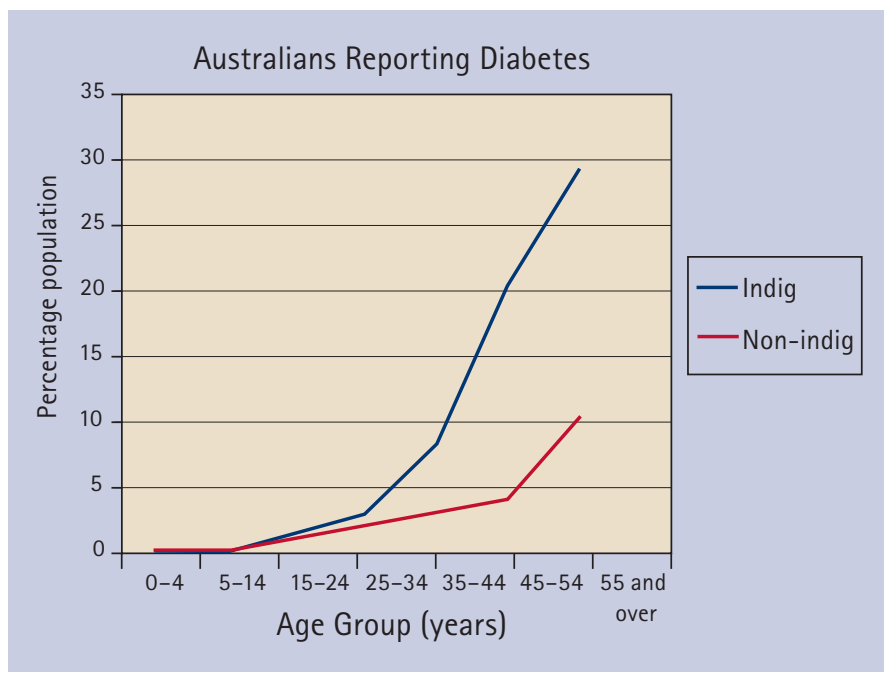

Fig. 4 \% Persons Reporting Diabetes, National Health Survey, Australian Bureau of Statistics, 2001

\section{Rheumatic fever}

Rheumatic fever is an inflammatory disease which primarily affects children aged six to 15 and may develop after an infection with streptococcal bacteria (streptococcal pharyngitis or scarlet fever). Recurrence of rheumatic fever is relatively common in the absence of low dose antibiotics especially during the first three to five years following the initial episode. Complications of rheumatic fever can include permanent damage to the heart valves causing mitral/aortic stenosis.

Patients who have had rheumatic fever can contract bacterial endocarditis from oral bacteria which include streptococci, staphylococci, enterococci and candida albicans. This is most common through dental procedures which can introduce oral bacteria into the blood stream. Patients with a known history of rheumatic fever require antibiotic prophylaxis to protect them from bacterial endocarditis.

Although the quoted incidence rates for rheumatic fever among indigenous populations vary, figures show that they are among the highest in the world. As acute rheumatic fever can also frequently be misdiagnosed or under-reported, its true incidence is likely to be underestimated. ${ }^{27}$

In 2002, in the 'Top End' of the Northern Territory of Australia, the prevalence of rheumatic heart disease was just under 17 per 1,000 among Aboriginal and Torres Strait Islanders and less than two per 1,000 among other Australians. In Central Australia, the prevalence among Aboriginal and Torres Strait Islanders was 13 per 1,000 compared with less than one per 1,000 among other Australians living in the region ${ }^{27}$ (Fig. 5).

In 2001-2, indigenous groups of Australia were six to eight times as likely to be hospitalised for acute rheumatic fever and rheumatic heart disease and nearly 20 times as likely to die from these diseases as other Australians. ${ }^{27}$

Among indigenous peoples receiving heart valve surgery for acute rheumatic fever or rheumatic heart disease, nearly $45 \%$ are less than 25 years of age. In contrast, just 4\% of these procedures were performed on other Australians of a similar age. ${ }^{27}$

A thorough medical history is always required before treating any patient. However, the overseas dental practitioner working abroad in Australia must be made aware of the high prevalence of rheumatic fever among Aboriginal and Torres Strait Islander peoples to ensure that prophylactic precautions can be taken before dental procedures are commenced.

Dental practitioners should also be aware that dosage guidelines for antibiotic prophylaxis against bacterial endocarditis in Australia may differ from those stated in their home country. The Austral-

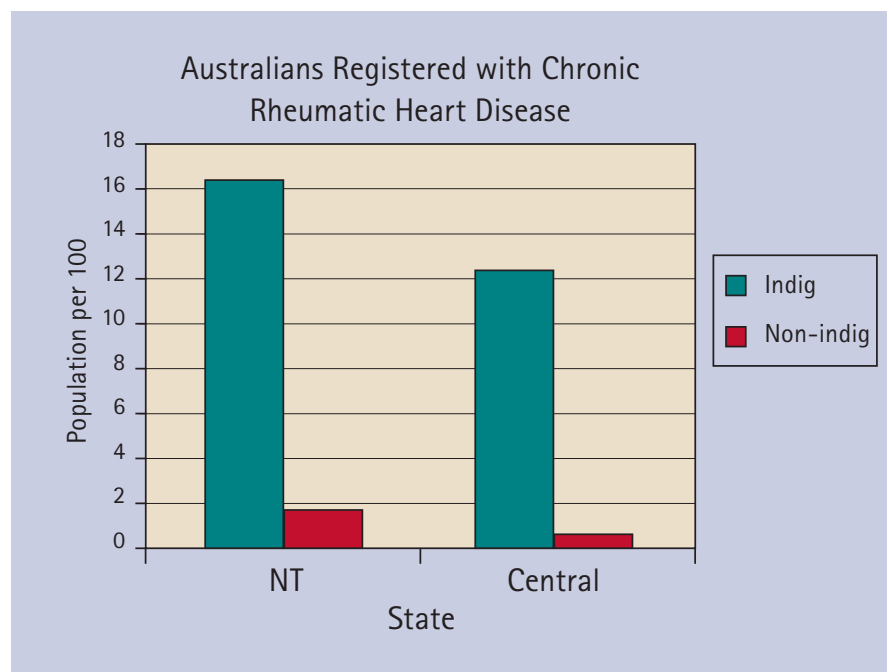

Fig.5 Population per 1000 in NT and Central Australia registered with chronic rheumatic heart disease in 2002 
ian Dental Association recommends as a standard, $2 \mathrm{~g}$ amoxicillin orally 1 hour pre-operatively and the overseas dental practitioner should be aware of this. ${ }^{28}$ Another consideration prior to providing antibiotic cover is whether the patient is on warfarin. The appropriate institutional guidelines should be followed in this case.

\section{Cultural awareness}

\section{The indirect approach}

Aboriginal people tend to prefer a less direct approach to communication and they may find cultures who prefer a direct/clear style somewhat confronting.

The customary way of seeking information is to establish a two way exchange, volunteering information of their own and hinting at what they would like to find out ${ }^{29}$.

\section{Indirect questioning}

While direct questions are used in Aboriginal society to determine background information for example where a person is from, detailed or personal information is sought through indirect questioning.

\section{Importance of non-verbals}

As discussed, some cultures rely heavily on the spoken word and the directness or preciseness that goes with it. Aboriginal culture is one where shared experiences, non-verbal and sensitivity to the context or circumstances contribute to an indirect style.

It is therefore important to pay considerable attention to nonverbals in order to pick up some of the reasons behind what is said. Observation and inference are the keys to understanding 'indirect interaction'.

\section{Silence}

In some non-indigenous cultures, silence in the communication process is seen as a gap that must be immediately filled.

In Aboriginal society, lengthy periods of silence are the 'norm' and are expected during conversation, particularly during information sharing and information seeking. Aboriginal people use silence to listen, allow for consensus or to indicate non-committal. The positive use of silence in Aboriginal interaction should never be interpreted as a lack of understanding.

\section{Eye contact}

Another important aspect of cross cultural communication is eye contact. In some cultures if we fail to maintain eye contact we can be perceived as 'hiding something' or someone not to be trusted. However, generally speaking use of indirect eye contact in Aboriginal culture implies respect.

Direct eye contact with anyone other than your most intimate peers or relations is seen as a sign of rudeness, disrespect or even aggression, and the appropriate strategy to convey polite respect is to avert or lower your eyes in conversation.

\section{Time}

What importance cultures places around time also affects the communication process. Some cultures are more orientated to the preciseness of 'getting the job done' and follow prescribed schedules. However, other cultures place far more significance on the interpersonal and as such are more flexible when it comes to schedules.

Communicating with Indigenous groups may require more time and patience as there are often considerable time delays between initiating dialogue and receiving feedback.

\section{Agreeing (saying yes)}

The tendency to agree with someone, regardless of whether you actually agree with them is a very common feature of Aboriginal conversation. It is customarily used to indicate a readiness for co-operative interaction, or resignation to the futility of the situation.

\section{CONCLUSIONS}

The indigenous Australian community is a high risk population for dental caries and periodontal disease. Poor oral hygiene and a diet high in refined carbohydrate are direct contributing factors. The widespread prevalence of NIDDM, which can be related to obesity and a diet high in fat and sugar, has been linked to the increases seen in periodontal disease and dental caries. This situation, combined with the high prevalence of rheumatic fever among the indigenous population, illustrates just how unique the problem for dental practitioners working in Australia is.

The oral health of the Australian indigenous population has been recognised as one of the key priority health issues by the National Aboriginal Torres Strait Islander Health Council (NATSI$\mathrm{HC})$. Along with an increase in funding, resources and recruitment of oral health professionals, a recommendation has been made that all oral health providers undertake cultural awareness training so that culturally appropriate oral health services are provided whether delivered by Aboriginal community controlled health services, government or private practice.

There was also a recommendation to integrate oral health into education, health and disease strategies so that the relationship of oral health with other health issues, such as diabetes and rheumatic fever, is strengthened.

On an individual level to the dental practitioner considering to practice in Australia, this brief review is aimed to give an overview of indigenous Australian health and how it relates specifically to oral health. Effective cross cultural skills are also critical to the provision of culturally sensitive oral health care.

Many thanks to Lyn Hart from Dentist Job Search and Sharon Marinucci from Australian Health Sector Recruitment for the information provided to help write this article.

1. Australian Bureau of Statistics. Population distribution, indigenous Australians. In Census 2001. pp 5-14. Sydney: Australian Government Press, 2001.

2. Sanderson A T. Notes on some skeletal changes in pre-European contact Australian Aborigines. J Hum Evol 1980; 2: 52-54.

3. Wall C H. Oral health status and tradition in Australia. Int Dent J 1984; 34: 271-277.

4. Franklin M, White L. The history and politics of Aboriginal health. In Ried J, Trompl $P$ (Eds). The health of Aboriginal Australia. Pp 1-23. Sydney: Harcourt, Brace and Javanovich, 1991

5. Kailis D J. Partial fluoridation and reduction of dental caries in Groote Eylanders Aboriginal children 1974-78. Aboriginal Health Info 1982; 24: 25-30.

6. Monk M. The health worker and the care of teeth. Aboriginal Health Worker 1978; 2: 52-54.

7. Davies M J, Spencer A J, Westwater A, Simmons B. Dental caries among Australian Aboriginal, non-Aboriginal Australian born, and overseas born children. Bulletin WHO 1997; 75: 197-203.

8. Seow W K, Amaratunge A, Sim R, Wan A. Prevalence of caries in urban Australian Aborigines aged 1-3.5 years. Paed Dent 1999; 21:91-96.

9. Regional Health Survey 1993. Food Habits, Food Inadequacy and Overweight, Epidemiology and Health Information Branch, Queensland Health.

10. Gracey M. Under nutrition in the midst of plenty: nutritional problems of young Aboriginals. Aust Paediatr J 1976; 12: 180-182.

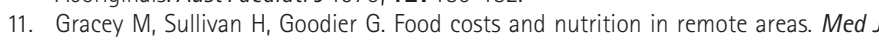
Aust 1991; 154: 294

12. Akikusa J D, Garrick D, Nash M C. Scurvy: forgotten but not gone. J Paediatr Child Health 2003; 39: 75-77.

13. Aboriginal and Torres Strait Islander Health Policy 1994, Queensland Health.

14. DeFronzo R A. The triumvirate: beta cell, muscle, liver. A collusion responsible for NIDDM. Diabetes 1988; 37: 667-687.

15. Endean C, Roberts-Thompson K, Wooley S. Anangu oral health: The status of the Indigenous population of the Anangu Pitjantjatjara lands. Aust J Rural Health 2004; 12: 99.

16. Oliver R C, Tervonen T. Diabetes - A risk factor for periodontitis in adults? J Periodontol 1994; 65: 530-538

17. Dennison D K, Gottsegen R, Rose L F. Diabetes and periodontal diseases. J Periodonto 1996; 67: 166-176

18. Cerda J, Vasquez de la Torre C, Malacara J M, Nava L E. Periodontal disease in noninsulin dependent diabetes mellitus (NIDDM). The effect of age and time since 
diagnosis. J Periodontol 1994; 65: 991-995.

19. Taylor $\mathrm{G} W$ et al. Non-insulin dependent diabetes mellitus and alveolar bone loss progression over 2 years. J Periodontol 1998; 69: 76-83.

20. Dorocka-Bobkowska B, Budtz-Jorgenson E, Wloch S. Non-insulin dependent diabetes mellitus as a risk factor for denture stomatitis. J Oral Pathol Med 1996; 25: 411-415.

21. Collin $\mathrm{H} L$ et al. Oral symptoms and signs in elderly patients with type 2 diabetes. A focus on diabetic neuropathy. Oral Surg Med Pathol Rad Endodont 2000; 90: 299305.

22. Guest C S, O'Dea K. Diabetes in Aborigines and other Australian populations. Aust $J$ Public Health 1992; 16: 340-349.

23. Daniel $\mathrm{M}$ et al. Diabetes Incidence in an Australian Aboriginal Population. Diabetes Care 1999; 22: 1993-1999
24 O'Dea K Patel M, Kubish D et al Obesity, diabetes, and hyperlipidaemia in a central Australian Aboriginal community with longstanding history of acculturation Diabetes Care 1993; 16: 1004-1010.

25. Leonard $\mathrm{D}$ et al. Obesity, diabetes and associated cardiovascular risks among Torres Strait Islander people. Aust N Z J Pub Health 2002; 26: 144-149.

26. National Aboriginal and Torres Strait Islander Oral Health Workshop. Workshop Report and Action Plan. Commonwealth Department of Health and Ageing, Canberra, 2003.

27. Rheumatic heart disease: all but forgotten in Australia except among Aboriginal and Torres Strait Islander peoples. Australian Institute of Health and Welfare, 2004

28. Spicer J. Therapeutic Guidelines: antibiotics. (11th edn.) Melbourne: Therapeutic Guidelines, 2000

29. Aboriginal and Torres Strait Islander Cultural Awareness Program. Queensland Health. 2003 